\title{
Bounds on the Minimal Energy of Translation Invariant $N$-Polaron Systems
}

\author{
Marcel Griesemer \\ Universität Stuttgart, Fachbereich Mathematik \\ 70550 Stuttgart, Germany \\ email: marcel@mathematik.uni-stuttgart.de \\ Jacob Schach Møller \\ Aarhus University, Department of Mathematical Sciences \\ 8000 Århus C, Denmark \\ email: jacob@imf.au.dk
}

\begin{abstract}
For systems of $N$ charged fermions (e.g. electrons) interacting with longitudinal optical quantized lattice vibrations of a polar crystal we derive upper and lower bounds on the minimal energy within the model of H. Fröhlich. The only parameters of this model, after removing the ultraviolet cutoff, are the constants $U>0$ and $\alpha>0$ measuring the electron-electron and the electron-phonon coupling strengths. They are constrained by the condition $\sqrt{2} \alpha<U$, which follows from the dependence of $U$ and $\alpha$ on electrical properties of the crystal. We show that the large $N$ asymptotic behavior of the minimal energy $E_{N}$ changes at $\sqrt{2} \alpha=U$ and that $\sqrt{2} \alpha \leq U$ is necessary for thermodynamic stability: for $\sqrt{2} \alpha>U$ the phonon-mediated electron-electron attraction overcomes the Coulomb repulsion and $E_{N}$ behaves like $-N^{7 / 3}$.
\end{abstract}




\section{Introduction}

We study a system of $N$ electrons in a polar (ionic) crystal, modelled by a Hamiltonian derived by H. Fröhlich [12]. The model takes into account the electron-electron Coulomb repulsion, and a linear interaction of the electrons with the longitudinal optical phonons. The model is called the 'large polaron' model, since it assumes that a polaron (dressed electron) extends over a region which is large compared to the ion-ion spacing. In particular the underlying discrete (and infinite) crystal is replaced by a continuum. See [7, 11, 19].

As is well-known, linear electron-phonon couplings induce an effective pair attraction between electrons. This attraction competes with the electron-electron repulsion and may cause a phase-transition as the electron-phonon interaction strength increases. This mechanism is behind the production of Cooper pairs in the BCS model of low temperature superconductivity, and in high- $T_{\mathrm{c}}$ superconductivity the role of many-polaron systems is being investigated [1, 8, 17].

The Fröhlich Hamiltonian depends on two non-negative dimensionless quantities, $U$ and $\alpha$. The constant $U$ is the electron-electron repulsion strength, and $\alpha$ is the Fröhlich electronphonon coupling constant. Physically relevant models must satisfy the constraint, cf. [4, 28],

$$
\sqrt{2} \alpha<U
$$

In this paper we prove upper and lower bounds on the minimal energy $E_{N}$ of the $N$ electron Fröhlich Hamiltonian for all $N$ and all non-negative values of $U, \alpha$. In the unphysical regime $\sqrt{2} \alpha \geq U$, our results imply that $E_{N} \sim-N^{7 / 3}$. In the physical regime we find that $E_{N} \geq-C N^{2}$, thus establishing a sharp transition in the large $N$-asymptotics of $E_{N}$ at $\sqrt{2} \alpha=U$. This transition is due to the mediated attraction between electrons overcoming the repulsion at $\sqrt{2} \alpha=U$ in the limit of large $N$. In fact, the quantity $U-\sqrt{2} \alpha$ appears in our analysis as an effective Coulomb coupling strength. We also demonstrate that $E_{N} \leq-\alpha N$ and $E_{N+M} \leq E_{N}+E_{M}$ in the physical regime. We do not know whether or not $E_{N}$ is an extensive quantity, but if it is not extensive, then this must be due to electron-phonon correlations, cf. Proposition A.3

We pause this discussion to introduce the mathematical model. The Fröhlich Hamiltonian describing $N$ electrons in a polar crystal reads

$$
\sum_{\ell=1}^{N}\left[-\frac{1}{2} \Delta_{x_{\ell}}+\sqrt{\alpha} \Phi\left(x_{\ell}\right)\right]+H_{\mathrm{ph}}+U V_{\mathrm{C}},
$$

where the number operator

$$
H_{\mathrm{ph}}=\int_{\mathbb{R}^{3}} a^{*}(k) a(k) d k,
$$

accounts for the kinetic energy of the phonons while the field operator

$$
\Phi(x)=\int_{\mathbb{R}^{3}} \frac{1}{c_{0}|k|}\left[e^{1 k \cdot x} a(k)+e^{-1 k \cdot x} a^{*}(k)\right] d k,
$$

is responsible for the electron-phonon interaction. Here $c_{0}:=2^{3 / 4} \pi$. Finally the electronelectron interaction is given by the sum of two-body Coulomb potentials

$$
V_{\mathrm{C}}\left(x_{1}, \ldots, x_{N}\right)=\sum_{1 \leq i<j \leq N} \frac{1}{\left|x_{i}-x_{j}\right|} .
$$


We work in units where the frequency of the longitudinal optical phonons, $\omega_{\mathrm{LO}}$, Planck's constant $\hbar$, and the electron band mass are equal to one.

Let $\mathcal{F}$ denote the symmetric Fock space over $L^{2}\left(\mathbb{R}^{3}\right)$. The Hamiltonian (1.1) defines a symmetric quadratic form on $\mathcal{H}=\wedge^{N} L^{2}\left(\mathbb{R}^{3}\right) \otimes \mathcal{F}$, but, a priori, it is not well defined as a selfadjoint operator. For that one must first impose an ultraviolet cutoff on the electron-phonon interaction: Let $\Lambda>0$, and define the cutoff Hamiltonian as

$$
H_{N, \Lambda}=\sum_{\ell=1}^{N}-\frac{1}{2} \Delta_{x_{\ell}}+H_{\mathrm{ph}}+\sqrt{\alpha} \sum_{\ell=1}^{N} \Phi_{\Lambda}\left(x_{\ell}\right)+U V_{\mathrm{C}}
$$

where

$$
\Phi_{\Lambda}(x)=\int_{|k| \leq \Lambda} \frac{1}{c_{0}|k|}\left[e^{1 k \cdot x} a(k)+e^{-1 k \cdot x} a^{*}(k)\right] d k .
$$

The operators $H_{N, \Lambda}$ are self-adjoint on $\mathcal{D}\left(H_{\mathrm{ph}}\right) \cap \mathcal{D}\left(\sum_{\ell=1}^{N} \Delta_{x_{\ell}}\right)$, by the Kato-Rellich theorem, and it is well known, cf. [2, 6, 13, 14, 27], that $H_{N, \Lambda}$ converges, as $\Lambda \rightarrow \infty$, in the normresolvent sense to a self-adjoint operator, which we denote by $H_{N}$. This implies that

$$
E_{N}=\lim _{\Lambda \rightarrow \infty} E_{N, \Lambda}
$$

if $E_{N, \Lambda}:=\inf \sigma\left(H_{N, \Lambda}\right)$ and $E_{N}:=\inf \sigma\left(H_{N}\right)$.

The main goal of this paper is to investigate the large $N$ behavior of the minimal energy $E_{N}$ as a function of $\alpha$ and $U$. Our first result is an upper bound in the regime $\sqrt{2} \alpha>U$.

Theorem 1.1. There is a constant $C$ such that for all $N$ and for $\sqrt{2} \alpha \geq U \geq 0$

$$
E_{N} \leq(\sqrt{2} \alpha-U)^{2} N^{\frac{7}{3}}\left[E_{\mathrm{PTF}}+C N^{-\frac{1}{17}}\right] .
$$

Here $E_{\mathrm{PTF}}<0$ is given by (1.3) below.

Theorem 1.1 is proved variationally by using Pekar's ansatz in terms of a product state, which is known to give the correct ground state energy for $N=1,2$ in the large $\alpha$ limit [9, 25, 26]. Taking the expectation value in a state $f \otimes \eta \in \wedge^{N} L^{2}\left(\mathbb{R}^{3}\right) \otimes \mathcal{F}$ and explicitly minimizing with respect to $\eta$ we arrive at a Hartree-Fock type energy which is then estimated by a Thomas-Fermi energy. This allows us to scale out all parameters and we are left with the bound in Theorem 1.1 , where

$$
\begin{gathered}
E_{\mathrm{PTF}}=\inf _{\rho \geq 0, \int \rho(x) d x=1} \mathcal{E}_{\mathrm{PTF}}(\rho), \\
\mathcal{E}_{\mathrm{PTF}}(\rho):=\frac{3}{10}\left(6 \pi^{2}\right)^{\frac{2}{3}} \int_{\mathbb{R}^{3}} \rho(x)^{\frac{5}{3}} d x-\frac{1}{2} \int_{\mathbb{R}^{6}} \frac{\rho(x) \rho(y)}{|x-y|} d x d y .
\end{gathered}
$$

We note that in the error term in Theorem 1.1 the exponent $1 / 17$ can be replaced by any number less than $2 / 33$ at the expense of a larger and divergent constant $C$.

To show that the variational upper bound from Theorem 1.1 has the right asymptotics in $N$ and $\alpha$, we provide the following lower bound:

Theorem 1.2. There exists $C>0$ such that for all $N$ and $\sqrt{2} \alpha \geq U \geq 0$,

$$
E_{N} \geq-C_{\mathrm{G}}(\sqrt{2} \alpha-U)^{2} N^{\frac{7}{3}}-C \alpha^{2} N^{\frac{7}{3}-\frac{1}{9}} .
$$


This lower bound is obtained, essentially, by completing the square with respect to creation and annihilation operators in the expression $H_{\mathrm{ph}}+\sqrt{\alpha} \sum_{j=1}^{n} \Phi\left(x_{j}\right)$. The computation brings out an effective Coulomb interaction with coupling strength $-\sqrt{2} \alpha$. Unfortunately, it also yields an infinite self-energy, which must be dealt with before completing the square. For that we use a commutator argument from [25], which is responsible for the error term in Theorem 1.2 The resulting effective Hamiltonian with an attractive Coulomb potential is bounded below by the 'gravitational collapse' bound

$$
\sum_{j=1}^{N}-\frac{1}{2} \Delta_{j}-\sum_{1 \leq j<\ell \leq N} \frac{1}{\left|x_{j}-x_{\ell}\right|} \geq-C_{\mathrm{G}} N^{\frac{7}{3}}
$$

due to Lévy-Leblond [20, Theorem 2]. Hence the presence of the constant $C_{\mathrm{G}}$ in Theorem 1.2

We now turn to the physical regime $\sqrt{2} \alpha<U$. Here our lower bound is a byproduct of our proof of Theorem 1.2, and we have no reason to believe it is optimal. Together with Theorem 1.1 it demonstrates, however, that the model undergoes a sharp transition at $\alpha=$ $U / \sqrt{2}$.

Theorem 1.3. For $0<\sqrt{2} \alpha<U$,

$$
E_{N} \geq-\left(\frac{16}{3 \pi} \alpha^{2} N^{2}+3\right) \frac{U}{U-\sqrt{2} \alpha} .
$$

Last but not least there are the following universal variational upper bounds for $E_{N}$ and $E_{N+M}$.

Theorem 1.4. For all $N, M, \alpha$ and $U$ we have

$$
\begin{aligned}
E_{N} & \leq-\alpha N, \\
E_{N+M} & \leq E_{N}+E_{M} .
\end{aligned}
$$

The bound $E_{1} \leq-\alpha$ is well known from [19, 10] and it agrees with the result of a formal computation of $E_{1}$ by second order perturbation theory [11]. Also, it is consistent with Haga's computation of $E_{1}$ including $\alpha^{2}$-terms 1 [16]. The bound $E_{N} \leq-\alpha N$ follows from the estimates $E_{1} \leq-\alpha$ and $E_{N} \leq N E_{1}$, the latter of which is a consequence of the second result of Theorem 1.4. We remark that $E_{N+M} \leq E_{N}+E_{M}$ holds quite generally for translation invariant $N$-particle systems with interactions that go to zero with increasing particle separation. In particular it holds for fermions and for distinguishable particles alike. Numerically computed upper bounds on $E(N) / N$, for $N=2$ through $N=32$ can be found in the literature [5], but in the case of fermions they are not refined enough to be consistent with the bound $E_{N+M} \leq E_{N}+E_{M}$.

In this paper we have omitted spin, but the Fermi statistics is taken into account. There are only few small modifications necessary for treating fermions with $q$ spin states, such as factor of $q^{-2 / 3}$ in front of the Thomas-Fermi kinetic energy, which alters the upper bound in Theorem 1.1 by a factor of $q^{2 / 3}$.

The many-polaron model has also been studied with a confining potential of the form $\sum_{\ell=1}^{N} W\left(x_{\ell}\right), W(0)=0$ and $W \geq 0$ included in the Hamiltonian [18]. We could include such a potential in our work as well, but, at least in the regime $\sqrt{2} \alpha>U$ this would not affect the leading large $N$ behaviour of $E_{N}$.

\footnotetext{
${ }^{1}$ There is a sign error in Feynman's quote of Haga's result.
} 


\section{Upper bounds on $E_{N}$}

In this section we prove Theorem 1.1 and Theorem 1.4. Since $E_{N}=\lim _{\Lambda \rightarrow \infty} E_{N, \Lambda}$ we only need to deal with the self-adjoint operator $H_{N, \Lambda}$. Let $f \in \mathcal{D}_{N}=\wedge^{N} L^{2}\left(\mathbb{R}^{3}\right) \cap H^{1}\left(\mathbb{R}^{3 N}\right)$ be normalized and recall that the one-particle density matrix $\gamma$ and the density function $\rho$ associated with $f$ are defined by

$$
\begin{gathered}
\gamma\left(x, x^{\prime}\right):=N \int_{\mathbb{R}^{3(N-1)}} f\left(x, x_{2}, \ldots, x_{N}\right) \overline{f\left(x^{\prime}, x_{2}, \ldots, x_{N}\right)} d x_{2} \cdots d x_{N}, \\
\rho(x):=\gamma(x, x)=N \int_{\mathbb{R}^{3(N-1)}}\left|f\left(x, x_{2}, \ldots, x_{N}\right)\right|^{2} d x_{2} \cdots d x_{N} .
\end{gathered}
$$

In this paper the Fouriertransform $\hat{\rho}$ of the density function $\rho$, or of any other function, is defined by:

$$
\hat{\rho}(k)=\int_{\mathbb{R}^{3}} e^{-1 k \cdot x} \rho(x) d x,
$$

that is, without a factor of $(2 \pi)^{-3 / 2}$.

Proposition 2.1. Suppose $\sqrt{2} \alpha \geq U$. Then for every one-particle density matrix $\gamma$ on $L^{2}\left(\mathbb{R}^{3}\right)$ with $0 \leq \gamma \leq 1, \operatorname{Tr}[\gamma]=N, \operatorname{Tr}[-\Delta \gamma]<\infty$, and for $\rho(x):=\gamma(x, x)$,

$$
\begin{aligned}
E_{N} \leq & (\sqrt{2} \alpha-U)^{2}\left[\frac{1}{2} \operatorname{Tr}[-\Delta \gamma]-\frac{1}{2} \int_{\mathbb{R}^{6}} \frac{\rho(x) \rho(y)}{|x-y|} d x d y\right] \\
& -U(\sqrt{2} \alpha-U) \frac{1}{2} \int_{\mathbb{R}^{6}} \frac{|\gamma(x, y)|^{2}}{|x-y|} d x d y .
\end{aligned}
$$

Proof. This proof is based on the estimate $E_{N, \Lambda} \leq\left\langle f \otimes \eta, H_{N, \Lambda} f \otimes \eta\right\rangle$ for suitable normalized $f \in \mathcal{D}_{N}$ and $\eta \in \mathcal{F}$. We begin by observing that the expectation value of the interaction operator in a state $f \otimes \eta$ may be represented in the following two ways: if $f$ and $\eta$ are normalized, then

$$
\begin{aligned}
\langle f & \left.\otimes \eta, \sum_{\ell=1}^{N} \Phi_{\Lambda}\left(x_{\ell}\right) f \otimes \eta\right\rangle \\
& =\int_{\mathbb{R}^{3 N}}\left|f\left(x_{1}, \ldots, x_{N}\right)\right|^{2} \sum_{\ell=1}^{N} V_{\Lambda, \eta}\left(x_{\ell}\right) d x_{1} \ldots d x_{N} \\
& =\left\langle\eta, \Phi_{\Lambda}(\rho) \eta\right\rangle
\end{aligned}
$$

where $V_{\Lambda, \eta}(x):=\left\langle\eta, \Phi_{\Lambda}(x) \eta\right\rangle, \rho$ is the density associated with $f$, and

$$
\begin{aligned}
\Phi_{\Lambda}(\rho) & :=\int_{\mathbb{R}^{3}} \rho(x)\left\langle\eta, \Phi_{\Lambda}(x) \eta\right\rangle d x \\
& =\int_{|k| \leq \Lambda} \frac{1}{c_{0}|k|}\left[\hat{\rho}(k) a(k)+\hat{\rho}(k) a^{*}(k)\right] d k .
\end{aligned}
$$

Hence if we define $H_{N, \Lambda}^{\eta}:=\sum_{\ell=1}^{N}\left[-\frac{1}{2} \Delta_{\ell}+\sqrt{\alpha} V_{\Lambda, \eta}\left(x_{\ell}\right)\right]+U V_{C}$, then

$$
\left\langle f \otimes \eta, H_{N, \Lambda} f \otimes \eta\right\rangle=\left\langle f, H_{N, \Lambda}^{\eta} f\right\rangle+\left\langle\eta, H_{\mathrm{ph}} \eta\right\rangle .
$$


The ground state energy of the $N$-body Hamiltonian $H_{N, \Lambda}^{\eta}$ is bounded above by its ground state energy in the Hartree-Fock approximation. By Lieb's variational principle, [23] and [3, Corollary 1], this Hartree-Fock ground state energy is bounded above by

$$
\mathcal{E}_{H F}^{N, \Lambda}(\gamma, \eta):=\operatorname{Tr}\left[\left(-\frac{1}{2} \Delta+\sqrt{\alpha} V_{\Lambda, \eta}\right) \gamma\right]+\frac{U}{2} \int_{\mathbb{R}^{6}} \frac{\rho(x) \rho(y)-|\gamma(x, y)|^{2}}{|x-y|} d x d y
$$

for any one-particle density matrix $\gamma$ with $\operatorname{Tr}[\gamma]=N$ and $\rho(x)=\gamma(x, x)$. Hence, in view of (2.5), we conclude that

$$
E_{N, \Lambda} \leq \mathcal{E}_{H F}^{N, \Lambda}(\gamma, \eta)+\left\langle\eta, H_{\mathrm{ph}} \eta\right\rangle
$$

for all normalized $\eta \in \mathcal{F}$. In order to minimize the right hand side with respect to $\eta$, we use that (2.3) equals (2.4). It follows, by Lemma A.2, that

$$
\inf _{\eta \in \mathcal{F},\|\eta\|=1}\left[\sqrt{\alpha} \operatorname{Tr}\left(V_{\Lambda, \eta} \gamma\right)+\left\langle\eta, H_{\mathrm{ph}} \eta\right\rangle\right]=-\frac{\alpha}{c_{0}^{2}} \int_{|k| \leq \Lambda} \frac{|\hat{\rho}(k)|^{2}}{|k|^{2}} d k .
$$

By combining (2.6), (2.7), and (2.8) and then letting $\Lambda \rightarrow \infty$ we arrive at

$$
E_{N} \leq \frac{1}{2} \operatorname{Tr}[-\Delta \gamma]+(U-\sqrt{2} \alpha) \frac{1}{2} \int_{\mathbb{R}^{6}} \frac{\rho(x) \rho(y)}{|x-y|} d x d y-\frac{U}{2} \int_{\mathbb{R}^{6}} \frac{|\gamma(x, y)|^{2}}{|x-y|} d x d y
$$

for any one-particle density matrix $\gamma$ with $\operatorname{Tr}(\gamma)=N$ and $\rho(x)=\gamma(x, x)$. Here (A.3) and (1.2) were used also. In the case $\sqrt{2} \alpha=U$ it is clear from (2.9) or from (2.5) with $\eta$ being the vacuum vector, that $E_{N} \leq 0$. In the case where $\beta:=\sqrt{2} \alpha-U>0$, we choose the density matrix $\gamma$ on the form $\gamma=U_{\beta} \widetilde{\gamma} U_{\beta}^{*}$ with $U_{\beta}$ defined by $\left(U_{\beta} \varphi\right)(x):=\beta^{3 / 2} \varphi(\beta x)$. The Proposition then follows from $U_{\beta}^{*} \Delta U_{\beta}=\beta^{2} \Delta$ and from $\gamma(x, y)=\beta^{3} \widetilde{\gamma}(\beta x, \beta y)$ by a simple change of variables in the integrals of (2.9).

The second ingredient for proving Theorem 1.1 is the following lemma.

Lemma 2.2. Let $g \in H^{2}\left(\mathbb{R}^{3}\right)$ with $\|g\|=1$. Then for every $\rho \in L^{1}\left(\mathbb{R}^{3}\right)$ with $\rho \geq 0$ and $\int_{\mathbb{R}^{3}} \rho(x) d x=N$ there exists a density matrix $\gamma$ such that $\gamma(x, x)=\left(\rho *|g|^{2}\right)(x)$ and

$$
\operatorname{Tr}[-\Delta \gamma]=\frac{3}{5}\left(6 \pi^{2}\right)^{\frac{2}{3}} \int_{\mathbb{R}^{3}} \rho(x)^{\frac{5}{3}} d x+N\|\nabla g\|^{2} .
$$

Proof. For the reader's convenience, we recall the proof from [22, Page 621]. Let $M: \mathbb{R}^{6} \rightarrow \mathbb{R}$ be defined by $M(p, q)=1$ if $|p| \leq(6 \pi)^{2 / 3} \rho(q)^{1 / 3}$ and $M(p, q)=0$ otherwise. Then

$$
\begin{aligned}
(2 \pi)^{-3} \int_{\mathbb{R}^{6}} M(p, q) d p d q & =\int_{\mathbb{R}^{3}} \rho(q) d q=N \\
(2 \pi)^{-3} \int_{\mathbb{R}^{6}} p^{2} M(p, q) d p d q & =\frac{3}{5}(6 \pi)^{\frac{2}{3}} \int_{\mathbb{R}^{3}} \rho(q)^{\frac{5}{3}} d q .
\end{aligned}
$$

We define $\gamma$ by

$$
\gamma=(2 \pi)^{-3} \int_{\mathbb{R}^{6}} M(p, q) \Pi_{p q} d p d q
$$

where $\Pi_{p q}$ is the rank one projection given by

$$
\Pi_{p q} \varphi=g_{p q} \int_{\mathbb{R}^{3}} \overline{g_{p q}(x)} \varphi(x) d x, \quad g_{p q}(x)=e^{1 p x} g(x-q) .
$$


It follows that $\gamma(x, x)=\int_{\mathbb{R}^{3}}|g(x-q)|^{2} \rho(q) d q$, and from

$$
\operatorname{Tr}\left[-\Delta \Pi_{p q}\right]=\left\|\nabla g_{p q}\right\|^{2}=p^{2}+\|\nabla g\|^{2}+2 p \cdot\langle g,-1 \nabla g\rangle
$$

and (2.10) we find the asserted expression for $\operatorname{Tr}[-\Delta \gamma]$.

Proposition 2.1 and Lemma 2.2 suggest the definition of a Polaron Thomas-Fermi functional by

$$
\mathcal{E}_{\mathrm{PTF}}(\rho):=\frac{3}{10}\left(6 \pi^{2}\right)^{\frac{2}{3}} \int_{\mathbb{R}^{3}} \rho(x)^{\frac{5}{3}} d x-\frac{1}{2} \int_{\mathbb{R}^{6}} \frac{\rho(x) \rho(y)}{|x-y|} d x d y,
$$

where $\rho \in L^{1}\left(\mathbb{R}^{3}\right) \cap L^{5 / 3}\left(\mathbb{R}^{3}\right)$ and $\rho \geq 0$. If $\rho_{N}(x):=N^{2} \rho\left(N^{1 / 3} x\right)$, then $\left\|\rho_{N}\right\|_{1}=N\|\rho\|_{1}$ and

$$
\mathcal{E}_{\mathrm{PTF}}\left(\rho_{N}\right)=N^{\frac{7}{3}} \mathcal{E}_{\mathrm{PTF}}(\rho) .
$$

Hence it suffices to consider densities $\rho$ with $\int \rho(x) d x=1$. Let

$$
E_{\mathrm{PTF}}:=\inf \left\{\mathcal{E}_{\mathrm{PTF}}(\rho) \mid \rho \geq 0, \int_{\mathbb{R}^{3}} \rho(x) d x=1\right\}
$$

which is finite by Lemma A.1

Lemma 2.3. $E_{\mathrm{PTF}}<0$.

Proof. Given $\rho \in L^{1}\left(\mathbb{R}^{3}\right) \cap L^{5 / 3}\left(\mathbb{R}^{3}\right)$ with $\rho \geq 0$ and $\int \rho d x=1$, let $\rho_{R}(x)=R^{-3} \rho\left(R^{-1} x\right)$. Then $\int_{\mathbb{R}^{3}} \rho_{R}(x) d x=1$ for all $R>0$ and

$$
\mathcal{E}_{\mathrm{PTF}}\left(\rho_{R}\right)=R^{-2} \frac{3}{10}\left(6 \pi^{2}\right)^{\frac{2}{3}} \int_{\mathbb{R}^{3}} \rho(x)^{\frac{5}{3}} d x-R^{-1} \frac{1}{2} \int_{\mathbb{R}^{6}} \frac{\rho(x) \rho(y)}{|x-y|} d x d y .
$$

This is negative for $R$ large enough.

Proof of Theorem 1.1. Let $g \in L^{2}\left(\mathbb{R}^{3}\right)$ be given by $g(x)=(2 \pi)^{-3 / 4} e^{-x^{2} / 4}$ and set $g_{\varepsilon}(x)=$ $\varepsilon^{-3 / 2} g(x / \varepsilon)$, so that $\left\|g_{\varepsilon}\right\|=1$ for all $\varepsilon>0$. Let $\beta=\sqrt{2} \alpha-U \geq 0$. If $\beta=0$ then $E_{N} \leq 0$ by Proposition 2.1. Hence it remains to consider the case $\beta>0$. Every density function $\rho_{N} \in L^{1}\left(\mathbb{R}^{3}\right)$ with $\left\|\rho_{N}\right\|_{1}=N$ is of the form $\rho_{N}(x)=N^{2} \rho\left(N^{1 / 3} x\right)$ with $\|\rho\|_{1}=1$. From Proposition 2.1 and Lemma2.2 combined it follows that

$$
\beta^{-2} E_{N} \leq \frac{3}{10}\left(6 \pi^{2}\right)^{\frac{2}{3}} \int_{\mathbb{R}^{3}} \rho_{N}(x)^{\frac{5}{3}} d x-\frac{1}{2} \int_{\mathbb{R}^{6}} \frac{\rho_{N, \varepsilon}(x) \rho_{N, \varepsilon}(y)}{|x-y|} d x d y+N\left\|\nabla g_{\varepsilon}\right\|^{2},
$$

where $\rho_{N, \varepsilon}=\rho_{N} *\left|g_{\varepsilon}\right|^{2}$. Suppose $1<\mu<6 / 5$ and let $f(k):=\widehat{|g|^{2}}=e^{-k^{2} / 2}$. Then $\widehat{\rho_{N, \varepsilon}}(k)=\widehat{\rho_{N}}(k) \mid \widehat{\left.g_{\varepsilon}\right|^{2}}(k)=\widehat{\rho_{N}}(k) f(\varepsilon k)$ and

$$
\sup _{k \neq 0} \frac{1-|f(k)|^{2}}{|k|^{\mu-1}} \leq 1
$$


By definition of $f$, by (2.13), and by Lemma A.1

$$
\begin{aligned}
& \int_{\mathbb{R}^{6}} \frac{\rho_{N}(x) \rho_{N}(y)}{|x-y|} d x d y-\int_{\mathbb{R}^{6}} \frac{\rho_{N, \varepsilon}(x) \rho_{N, \varepsilon}(y)}{|x-y|} d x d y \\
&= \frac{1}{2 \pi^{2}} \int_{\mathbb{R}^{3}}\left(1-|f(\varepsilon k)|^{2}\right) \frac{\left|\widehat{\rho_{N}}(k)\right|^{2}}{|k|^{2}} d k \\
& \quad= \frac{1}{2 \pi^{2}} \varepsilon^{\mu-1} \int_{\mathbb{R}^{3}} \frac{1-|f(\varepsilon k)|^{2}}{|\varepsilon k|^{\mu-1}} \frac{\left|\widehat{\rho_{N}}(k)\right|^{2}}{|k|^{3-\mu}} d k \\
& \quad \leq \frac{1}{2 \pi^{2}} \varepsilon^{\mu-1} \int_{\mathbb{R}^{3}} \frac{\left|\widehat{\rho_{N}}(k)\right|^{2}}{|k|^{3-\mu}} d k \\
& \quad=N^{2+\frac{\mu}{3}} \varepsilon^{\mu-1} 2(2 \pi)^{\mu-2} \frac{c_{\mu}}{c_{3-\mu}} \int_{\mathbb{R}^{6}} \frac{\rho(x) \rho(y)}{|x-y|^{\mu}} d x d y .
\end{aligned}
$$

Combining this estimate with (2.12), we see that

$$
\begin{aligned}
\beta^{-2} E_{N} \leq & N^{\frac{7}{3}} \mathcal{E}_{\mathrm{PTF}}(\rho)+N \varepsilon^{-2}\|\nabla g\|^{2} \\
& +N^{2+\frac{\mu}{3}} \varepsilon^{\mu-1}(2 \pi)^{\mu-2} \frac{c_{\mu}}{c_{3-\mu}} \int_{\mathbb{R}^{6}} \frac{\rho(x) \rho(y)}{|x-y|^{\mu}} d x d y
\end{aligned}
$$

for all $\rho \in L^{1}\left(\mathbb{R}^{3}\right)$ with $\|\rho\|_{1}=1$. If $\left\{\rho_{n}\right\} \subset L^{1}\left(\mathbb{R}^{n}\right)$ is a minimizing sequence, $\mathcal{E}_{\mathrm{PTF}}\left(\rho_{n}\right) \rightarrow$ $E_{\mathrm{PTF}}$ as $n \rightarrow \infty$, then $\left\|\rho_{n}\right\|_{5 / 3}$ is uniformly bounded by (A.1), and hence so is the term $\int \rho_{n}(x) \rho_{n}(y) /|x-y|^{\mu} d x d y$ for $\mu<6 / 5$. Therefore, in the limit $n \rightarrow \infty$, we obtain

$$
\beta^{-2} E_{N} \leq N^{\frac{7}{3}} E_{\mathrm{PTF}}+\frac{1}{4} N \varepsilon^{-2}+N^{2+\frac{\mu}{3}} \varepsilon^{\mu-1} C_{\mu}
$$

where the constant $C_{\mu}$ is finite for $\mu<6 / 5$ and $\|\nabla g\|^{2}=1 / 4$ was used. Upon optimizing with respect to $\varepsilon$ we arrive at

$$
\beta^{-2} E_{N} \leq N^{\frac{7}{3}} E_{\mathrm{PTF}}+N^{\frac{9+5 \mu}{3+3 \mu}} D_{\mu}
$$

with a new constant $D_{\mu}$. This bound with the choice $\mu=37 / 31<6 / 5$ proves Theorem 1.1

Proof of Theorem 1.4 We only need to prove that $E_{1} \leq-\alpha$. The bound $E_{N} \leq-\alpha N$ will then follow from $E_{N+M} \leq E_{N}+E_{M}$ as pointed out in the introduction.

Following Nelson [27] we introduce

$$
B_{\Lambda}:=\frac{\sqrt{\alpha}}{c_{0}} \int_{|k| \leq \Lambda} \frac{1}{1\left(1+\frac{k^{2}}{2}\right)|k|}\left[e^{i k \cdot x} a(k)+e^{-1 k \cdot x} a^{*}(k)\right] d k .
$$

Then

$$
e^{1 B_{\Lambda}} H_{1, \Lambda} e^{-1 B_{\Lambda}}=\frac{1}{2}\left(p^{2}+2 a^{*} \cdot p+2 p \cdot a+a^{2}+\left(a^{*}\right)^{2}+2 a^{*} a\right)+H_{\mathrm{ph}}-\alpha e_{\Lambda},
$$

where

$$
\begin{aligned}
a & :=\frac{\sqrt{\alpha}}{c_{0}} \int_{|k| \leq \Lambda} \frac{k}{\left(1+\frac{k^{2}}{2}\right)|k|} e^{1 k x} a(k) d k . \\
e_{\Lambda} & :=\frac{1}{c_{0}^{2}} \int_{|k| \leq \Lambda} \frac{1}{|k|^{2}\left(1+\frac{k^{2}}{2}\right)} d k
\end{aligned}
$$


From (2.14) we see that, for all normalized $f \in L^{2}\left(\mathbb{R}^{3}\right)$,

$$
\left\langle f \otimes \Omega, e^{1 B_{\Lambda}} H_{1, \Lambda} e^{-1 B_{\Lambda}} f \otimes \Omega\right\rangle=\left\langle f,\left(-\frac{1}{2} \Delta\right) f\right\rangle-\alpha e_{\Lambda}
$$

where $\Omega \in \mathcal{F}$ denotes the vacuum vector. Since $\inf \sigma(-\Delta)=0$ it follows from (2.15) that $E_{1, \Lambda} \leq-\alpha e_{\Lambda}$, where

$$
\lim _{\Lambda \rightarrow \infty} e_{\Lambda}=\frac{1}{c_{0}} \int_{\mathbb{R}^{3}} \frac{1}{|k|^{2}\left(1+\frac{k^{2}}{2}\right)} d k=1 .
$$

This concludes the proof of the first bound in Theorem 1.4

A result similar to $E_{N+M} \leq E_{N}+E_{M}$ is expressed by Theorem 6 in [15]. A copy of the proof of that theorem, with small modifications due to the differences of the Hamiltonians, also proves the desired bound here. In fact, the main part of the proof of [15, Theorem 6] is Equation (19) and the equation thereafter, which show that the interaction between electrons mediated by bosons decreases with increasing particle separation. This part remains valid for the coupling function $\chi_{|k| \leq \Lambda} /\left(c_{0}|k|\right)$ of the Hamiltonian $H_{N, \Lambda}$. Other parts of the proof are simplified due to the fact the phonon dispersion relation $\omega_{L O}$ is constant and hence a local operator with respect to the boson position as measured by $l_{k}$.

\section{Lower bounds on $E_{N}$}

In this section we prove Theorems 1.1, and 1.3. The first step is to make sure that phonons with large momenta contribute to lower order in $N$. To this end, for given $K, \Lambda, \delta, \kappa>0$, we define the operator

$$
\begin{aligned}
H_{N, \Lambda, K}:= & -\frac{1}{2}(1-\kappa) \sum_{\ell=1}^{N} \Delta_{\ell}+(1-\delta) H_{\mathrm{ph}}+U V_{\mathrm{C}} \\
& +\sqrt{\alpha} \sum_{\ell=1}^{N} \int_{|k| \leq \Lambda} \frac{e^{-\frac{|k|^{2}}{4 K^{2}}}}{c_{0}|k|}\left[e^{1 k \cdot x_{\ell}} a(k)+e^{-1 k \cdot x_{\ell}} a^{*}(k)\right] d k .
\end{aligned}
$$

Of course, later on, $\delta, \kappa \in(0,1)$ and $K<\Lambda \rightarrow \infty$. The following result, in the case $N=1$, is essentially due to Lieb and Thomas [25]. While a sharp cutoff $|k| \leq K$ is used in [25], we work with a Gaussian cutoff since we need the Fourier transform of the cutoff to be positive.

Lemma 3.1. Suppose $K, \Lambda, \alpha$ and $U$ are positive, $0<\delta<1$ and let $\kappa:=\frac{8 \alpha N}{3 K \delta} I_{\infty}$, where $I_{\infty}:=(\sqrt{2}-1) / \sqrt{\pi}$. Then

$$
H_{N, \Lambda} \geq H_{N, \Lambda, K}-\frac{3}{2 \delta}
$$

Proof. For each $\ell \in\{1, \ldots, N\}$, we introduce three high momenta modes by

$$
\begin{aligned}
Z_{j}^{(\ell)} & :=\int_{\mathbb{R}^{3}} \overline{T_{j}^{(\ell)}(k)} a(k) d k, \quad j \in\{1,2,3\}, \\
T_{j}^{(\ell)}(k) & :=\sqrt{\alpha} \chi_{\Lambda}(k) \frac{1-e^{-\frac{|k|^{2}}{4 K^{2}}}}{c_{0}|k|^{3}} k_{j} e^{-1 k \cdot x_{\ell}},
\end{aligned}
$$


$k_{j} \in \mathbb{R}$ being the $j$-th component of $k \in \mathbb{R}^{3}$ and $\chi_{\Lambda}$ the characteristic function of the set $\{|k| \leq$ $\Lambda$ \}. For later use we compute the inner product of two functions $T_{j}^{(\ell)}$. By straightforward computations,

$$
\int_{\mathbb{R}^{3}} \overline{T_{j}^{(\ell)}(k)} T_{j^{\prime}}^{(\ell)}(k) d k=\delta_{j j^{\prime}} \frac{\alpha}{3 K} I_{\frac{\Lambda}{K}},
$$

where

$$
I_{R}:=\frac{\sqrt{2}}{\pi} \int_{0}^{R} \frac{\left(1-e^{-\frac{s^{2}}{4}}\right)^{2}}{s^{2}} d s .
$$

Note that $4 \pi / c_{0}^{2}=\sqrt{2} / \pi$ and that $I_{\infty}=\lim _{R \rightarrow \infty} I_{R}=(\sqrt{2}-1) / \sqrt{\pi}$ as defined in the statement of the lemma. By definition of $H_{N, \Lambda, K}$,

$$
\begin{aligned}
H_{N, \Lambda} & =H_{N, \Lambda, K}+\sum_{\ell=1}^{N}\left(-\frac{\kappa}{2} \Delta_{\ell}+I_{K, \Lambda}^{(\ell)}\right)+\delta H_{\mathrm{ph}} \\
I_{K, \Lambda}^{(\ell)} & :=\sqrt{\alpha} \int_{|k| \leq \Lambda} \frac{1-e^{-\frac{|k|^{2}}{4 K^{2}}}}{c_{0}|k|}\left[e^{1 k \cdot x_{\ell}} a(k)+h . c .\right] d k
\end{aligned}
$$

where we introduced the operators $I_{K, \Lambda}^{(\ell)}$ associated with the ultraviolet part of the electronphonon interaction. The key ingredient of this proof is that

$$
I_{K, \Lambda}^{(\ell)}=\sum_{j=1}^{3}\left[p_{\ell, j}, Z_{j}^{(\ell)}-Z_{j}^{(\ell) *}\right]
$$

where $p_{\ell, j}:=-i \partial / \partial_{x_{\ell, j}}$. This identity implies that

$$
\begin{aligned}
\left|\left\langle\eta, I_{K, \Lambda}^{(\ell)} \eta\right\rangle\right| & \leq 2 \sum_{j=1}^{3}\left\|p_{\ell, j} \eta\right\|\left\|\left(Z_{j}^{(\ell)}-Z_{j}^{(\ell) *}\right) \eta\right\| \\
& \leq \frac{\kappa}{2}\left\langle\eta,-\Delta_{\ell} \eta\right\rangle+\frac{2}{\kappa} \sum_{j=1}^{3}\left\langle\eta,-\left(Z_{j}^{(\ell)}-Z_{j}^{(\ell) *}\right)^{2} \eta\right\rangle \\
& \leq \frac{\kappa}{2}\left\langle\eta,-\Delta_{\ell} \eta\right\rangle+\frac{4}{\kappa} \sum_{j=1}^{3}\left\langle\eta,\left(Z_{j}^{(\ell) *} Z_{j}^{(\ell)}+Z_{j}^{(\ell)} Z_{j}^{(\ell) *}\right) \eta\right\rangle,
\end{aligned}
$$

where $\kappa>0$ is to be selected, and the estimate

$$
\left|\left\langle\eta,\left(Z_{j}^{(\ell)}\right)^{2} \eta\right\rangle\right| \leq\left\|Z_{j}^{(\ell) *} \eta\right\|\left\|Z_{j}^{(\ell)} \eta\right\| \leq \frac{1}{2}\left\langle\eta,\left(Z_{j}^{(\ell)} Z_{j}^{(\ell) *}+Z_{j}^{(\ell) *} Z_{j}^{(\ell)}\right) \eta\right\rangle
$$

was used. From (3.2) and $I_{\Lambda / K} \leq I_{\infty}$ it is clear that

$$
\begin{aligned}
\sum_{j=1}^{3}\left(Z_{j}^{(\ell) *} Z_{j}^{(\ell)}+Z_{j}^{(\ell)} Z_{j}^{(\ell) *}\right) & =\sum_{j=1}^{3} 2 Z_{j}^{(\ell) *} Z_{j}^{(\ell)}+\left[Z_{j}^{(\ell)}, Z_{j}^{(\ell) *}\right] \\
& \leq \frac{2 \alpha I_{\infty}}{3 K} H_{\mathrm{ph}}+\frac{\alpha}{K} I_{\infty} .
\end{aligned}
$$

Combining (3.5) and (3.6) we arrive at

$$
\pm \sum_{\ell=1}^{N} I_{K, \Lambda}^{(\ell)} \leq \frac{\kappa}{2} \sum_{\ell=1}^{N}\left(-\Delta_{\ell}\right)+\frac{8 \alpha N I_{\infty}}{3 \kappa K} H_{\mathrm{ph}}+\frac{4 \alpha N I_{\infty}}{\kappa K},
$$

which, by (3.3) and the choice $\kappa=8 \alpha N I_{\infty} /(3 K \delta)$, proves the lemma. 
Lemma 3.2. Suppose $K, \Lambda, \alpha, U$ and $\kappa$ are positive, and $0<\delta \leq 1 / 2$. Then

$$
H_{N, \Lambda, K} \geq-\frac{1}{2}(1-\kappa) \sum_{\ell=1}^{N} \Delta_{\ell}-\left(\frac{\sqrt{2} \alpha}{1-\delta}-U\right) V_{\mathrm{C}}-\frac{2 \alpha N K}{\sqrt{\pi}} .
$$

Proof. By completing the square in annihilation and creation operators, that is, by using Lemma A.2, we see that

$$
\begin{aligned}
& (1-\delta) H_{\mathrm{ph}}+\frac{\sqrt{\alpha}}{c_{0}} \sum_{\ell=1}^{N} \int_{|k| \leq \Lambda} \frac{e^{-\frac{|k|^{2}}{4 K^{2}}}}{|k|}\left[e^{1 k \cdot x_{\ell}} a(k)+e^{-1 k \cdot x_{\ell}} a^{*}(k)\right] d k . \\
& \geq-\frac{\alpha}{(1-\delta) c_{0}^{2}} \sum_{j, \ell=1}^{N} \int_{\mathbb{R}^{3}} \frac{e^{-\frac{|k|^{2}}{2 K^{2}}}}{|k|^{2}} e^{1 k \cdot\left(x_{j}-x_{\ell}\right)} d k \\
& =-\frac{2 \alpha}{(1-\delta) c_{0}^{2}} \sum_{j<\ell} \int_{\mathbb{R}^{3}} \frac{e^{-\frac{|k|^{2}}{2 K^{2}}}}{|k|^{2}} e^{1 k \cdot\left(x_{j}-x_{\ell}\right)} d k-\frac{\alpha N K}{(1-\delta) \sqrt{\pi}} .
\end{aligned}
$$

The integral in (3.8) represents the electrostatic energy of two spherically symmetric, nonnegative charge distributions centered at $x_{j}$ and $x_{\ell}$, respectively, each distribution having total charge one, see (A.3). Hence Newton's theorem, [24, Theorem 9.7], implies that

$$
\int_{\mathbb{R}^{3}} \frac{e^{-\frac{|k|^{2}}{2 K^{2}}}}{|k|^{2}} e^{1 k \cdot\left(x_{j}-x_{\ell}\right)} d k \leq \frac{2 \pi^{2}}{\left|x_{j}-x_{\ell}\right|} .
$$

Since $c_{0}^{2}=2 \pi^{2} \sqrt{2}$, it follows that $(3.8)$ is bounded below by

$$
-\frac{\sqrt{2} \alpha}{1-\delta} V_{\mathrm{C}}-\frac{\alpha N K}{(1-\delta) \sqrt{\pi}}
$$

which proves the lemma.

Proof of Theorem 1.2. We shall combine the Lemmas 3.1 and 3.2 with suitable choices for $\delta$ and $K$. First, suppose that $0<\delta \leq 1 / 2$ and that $\kappa \in(0,1)$. Since $\sqrt{2} \alpha-U \geq 0$, by assumption of Theorem 1.2, the constant multiplying the potential $V_{\mathrm{C}}$ in Lemma 3.2 is positive, and hence, after the scaling transformation

$$
x \rightarrow\left(\frac{\sqrt{2} \alpha}{1-\delta}-U\right)^{-1}(1-\kappa) x
$$

we may apply (1.6) and find that

$$
\begin{aligned}
H_{N, \Lambda, K} & \geq-\frac{\left(\frac{\sqrt{2} \alpha}{1-\delta}-U\right)^{2}}{1-\kappa} \inf \sigma\left(\sum_{\ell=1}^{N}-\frac{1}{2} \Delta_{\ell}-V_{\mathrm{C}}\right)-\frac{2 \alpha N K}{\sqrt{\pi}} \\
& \geq-C_{\mathrm{G}} \frac{\left(\frac{\sqrt{2} \alpha}{1-\delta}-U\right)^{2}}{1-\kappa} N^{\frac{7}{3}}-\frac{2 \alpha N K}{\sqrt{\pi}}
\end{aligned}
$$

where $C_{\mathrm{G}}$ is chosen such that (1.6) holds true. 
We now make the choices

$$
\delta=\frac{1}{2} N^{-\frac{1}{9}} \text { and } K=\frac{1}{3} 32 I_{\infty} \alpha N^{1+\frac{2}{9}},
$$

which imply that $\kappa$, as defined in Lemma 3.1 obeys $\kappa=\frac{1}{2} N^{-1 / 9}=\delta$. Using that $(1-t)^{-1} \leq$ $1+2 t$, for $0 \leq t \leq 1 / 2$, that $U \leq \sqrt{2} \alpha$, and $I_{\infty} / \sqrt{\pi}=(\sqrt{2}-1) / \pi \leq 1 /(2 \pi)$, we find that

$$
\begin{aligned}
H_{N, \Lambda, K} & \geq-C_{\mathrm{G}}[\sqrt{2} \alpha(1+2 \delta)-U]^{2}(1+2 \kappa) N^{\frac{7}{3}}-\frac{32}{3 \pi} \alpha^{2} N^{2+\frac{2}{9}} \\
& \geq-C_{\mathrm{G}}\left[(\sqrt{2} \alpha-U)^{2}+16 \alpha^{2} \delta\right](1+2 \kappa) N^{\frac{7}{3}}-\frac{32}{3 \pi} \alpha^{2} N^{\frac{7}{3}-\frac{1}{9}} \\
& \geq-C_{\mathrm{G}}\left[(\sqrt{2} \alpha-U)^{2} N^{\frac{7}{3}}+18 \alpha^{2} N^{\frac{7}{3}-\frac{1}{9}}\right]-\frac{32}{3 \pi} \alpha^{2} N^{\frac{7}{3}-\frac{1}{9}} \\
& =-C_{\mathrm{G}}(\sqrt{2} \alpha-U)^{2} N^{\frac{7}{3}}-\left(18 C_{\mathrm{G}}+\frac{32}{3 \pi}\right) \alpha^{2} N^{\frac{7}{3}-\frac{1}{9}}
\end{aligned}
$$

Proof of Theorem 1.3. Finally we consider the case, where $U-\sqrt{2} \alpha>0$. In Lemma 3.2 we choose $\delta=(U-\sqrt{2} \alpha) /(2 U)$ and $K=8 \alpha N I_{\infty} /(3 \delta)$, so that $\kappa=1$ in Lemma 3.1, and

$$
U-\frac{\sqrt{2} \alpha}{1-\delta}=\frac{U-\sqrt{2} \alpha}{2(1-\delta)}>0 .
$$

From Lemma 3.1 and Lemma 3.2 it hence follows that

$$
E_{N} \geq-\frac{2 \alpha N K}{\sqrt{\pi}}-\frac{3}{2 \delta}=-\frac{16 I_{\infty}}{3 \sqrt{\pi}} \alpha^{2} N^{2} \frac{2 U}{U-\sqrt{2} \alpha}-\frac{3 U}{U-\sqrt{2} \alpha},
$$

where $I_{\infty} / \sqrt{\pi}=(\sqrt{2}-1) / \pi \leq 1 /(2 \pi)$.

\section{A Auxiliary Results}

Lemma A.1. Suppose that $\rho \in L^{1}\left(\mathbb{R}^{3}\right) \cap L^{5 / 3}\left(\mathbb{R}^{3}\right), \rho \geq 0,0<\mu<6 / 5$, and let $\rho_{N}(x)=$ $N^{2} \rho\left(N^{1 / 3} x\right)$. Then

$$
\begin{aligned}
\int_{\mathbb{R}^{6}} \frac{\rho(x) \rho(y)}{|x-y|^{\mu}} d x d y & \leq a_{\mu}\|\rho\|_{1}^{2-\frac{5 \mu}{6}}\|\rho\|_{\frac{5}{3}}^{\frac{5 \mu}{6}} \\
\int_{\mathbb{R}^{6}} \frac{\rho(x) \rho(y)}{|x-y|^{\mu}} d x d y & =(2 \pi)^{-\mu} \frac{c_{3-\mu}}{c_{\mu}} \int_{\mathbb{R}^{3}} \frac{|\hat{f}(k)|^{2}}{|k|^{3-\mu}} d k \\
\int_{\mathbb{R}^{6}} \frac{\rho(x) \rho(y)}{|x-y|} d x d y & =\frac{1}{2 \pi^{2}} \int_{\mathbb{R}^{3}} \frac{|\hat{f}(k)|^{2}}{|k|^{2}} d k \\
\int_{\mathbb{R}^{6}} \frac{\rho_{N}(x) \rho_{N}(y)}{|x-y|^{\mu}} d x d y & =N^{2+\frac{\mu}{3}} \int_{\mathbb{R}^{6}} \frac{\rho(x) \rho(y)}{|x-y|^{\mu}} d x d y
\end{aligned}
$$

where

$$
a_{\mu}:=\left(\frac{4 \pi}{3}\right)^{\frac{\mu}{3}}\left(\frac{6}{5 \mu}\right)^{1+\frac{\mu}{3}}\left(\frac{6}{5 \mu}-1\right)^{-1+\frac{\mu}{2}}, \quad c_{\mu}:=\pi^{-\frac{\mu}{2}} \Gamma\left(\frac{\mu}{2}\right)
$$

in (A.1) and (A.2), respectively. 
Inequality (A.1), in the special case $\mu=1$, implies that $\mathcal{E}_{\mathrm{PTF}}(\rho)$ is bounded below, and moreover, that $\|\rho\|_{5 / 3}$ is bounded unformly for densities $\rho$ with $\|\rho\|_{1}=1$ and $\mathcal{E}_{\mathrm{PTF}}(\rho) \leq$ $E_{\mathrm{PTF}}+1$.

Proof of Lemma $A .1$ For each $R>0$, by Hölder's inequality,

$$
\begin{aligned}
\int_{\mathbb{R}^{3}} \frac{\rho(y)}{|x-y|^{\mu}} d y & =\int_{|x-y| \leq R} \frac{\rho(y)}{|x-y|^{\mu}} d y+\int_{|x-y| \geq R} \frac{\rho(y)}{|x-y|^{\mu}} d y \\
& \leq\left(\frac{8 \pi}{6-5 \mu}\right)^{\frac{2}{5}} R^{\frac{6}{5}-\mu}\|\rho\|_{\frac{5}{3}}+R^{-\mu}\|\rho\|_{1} .
\end{aligned}
$$

By optimizing this bound w.r.to $R>0$ we obtain (A.1). Equation A.2 follows from [24, Corollary 5.10]. The factor $(2 \pi)^{-\mu}$ stems from the differences in the definition of the Fouriertransform. Equation A.3 is the important special case $\mu=1$ from (A.2), and A.4 is straightforward to verify by a change of variables.

Lemma A.2. Suppose $f \in L^{2}\left(\mathbb{R}^{3}\right)$. Then, for every $\delta>0$,

$$
\int_{\mathbb{R}^{3}}\left[\delta a^{*}(k) a(k)+\overline{f(k)} a(k)+f(k) a^{*}(k)\right] d k \geq-\frac{1}{\delta}\|f\|^{2}
$$

and the lower bound is attained by the expectation value in the coherent state $\eta \in \mathcal{F},\|\eta\|=1$, defined by $a(k) \eta=-\delta^{-1} f(k) \eta$.

Proof. By completeing the square in creation and annihilation operators

$$
\begin{aligned}
\int_{\mathbb{R}^{3}} & {\left[\delta a^{*}(k) a(k)+\overline{f(k)} a(k)+f(k) a^{*}(k)\right] d k } \\
= & \int_{\mathbb{R}^{3}}\left[\delta\left(a^{*}(k)+\frac{\overline{f(k)}}{\delta}\right)\left(a(k)+\frac{f(k)}{\delta}\right)-\frac{|f(k)|^{2}}{\delta}\right] d k \\
\geq & -\frac{1}{\delta}\|f\|^{2}
\end{aligned}
$$

Proposition A.3. Suppose that $\sqrt{2} \alpha \leq U$. Then for all $N, \Lambda>0$, and all $f \in \wedge^{N} L^{2}\left(\mathbb{R}^{3}\right), \eta \in$ $\mathcal{F}$ with $\|f\|=\|\eta\|=1$,

$$
\left\langle f \otimes \eta, H_{N, \Lambda} f \otimes \eta\right\rangle \geq-c_{\mathrm{L}} \frac{40}{3}\left(\frac{2}{3 \pi}\right)^{\frac{2}{3}} N,
$$

where $c_{\mathrm{L}}=1.68$ or any other constant for which the Lieb-Oxford inequality holds.

Proof. As in the proof of Theorem 1.1

$$
\begin{aligned}
\langle f & \left.\otimes \eta, H_{N, \Lambda} f \otimes \eta\right\rangle \\
& =\left\langle f,\left(-\frac{1}{2} \Delta+U V_{\mathrm{C}}\right) f\right\rangle+\left\langle\eta,\left(H_{\mathrm{ph}}+\sqrt{\alpha} \phi_{\Lambda}(\rho)\right) \eta\right\rangle \\
& \geq\left\langle f,\left(-\frac{1}{2} \Delta+U V_{\mathrm{C}}\right) f\right\rangle-\sqrt{2} \alpha \frac{1}{2} \int_{\mathbb{R}^{6}} \frac{\rho(x) \rho(y)}{|x-y|} d x d y .
\end{aligned}
$$


Using the Lieb-Thirring [24, Theorem 2.15] and the Lieb-Oxford inequalities [22] we find that

$$
\begin{aligned}
& \left\langle f \otimes \eta, H_{N, \Lambda}(f \otimes \eta)\right\rangle \\
& \quad \geq c_{\mathrm{LT}} \int_{\mathbb{R}^{3}} \rho(x)^{\frac{5}{3}} d x+(U-\sqrt{2} \alpha) \frac{1}{2} \int_{\mathbb{R}^{6}} \frac{\rho(x) \rho(y)}{|x-y|} d x d y-c_{\mathrm{L}} \int_{\mathbb{R}^{3}} \rho(x)^{\frac{4}{3}} d x
\end{aligned}
$$

where $c_{\mathrm{LT}}=\frac{3}{10}\left(\frac{3 \pi}{2}\right)^{2 / 3}$ and $c_{\mathrm{L}}=1.68$ or any other constant for which the Lieb-Oxford inequality is satisfied. From $\rho(x)^{4 / 3}=\rho(x)^{5 / 6} \rho(x)^{1 / 2}$ and the Cauchy-Schwarz inequality, for every $\varepsilon>0$,

$$
\begin{aligned}
\int_{\mathbb{R}^{3}} \rho(x)^{\frac{4}{3}} d x & \leq\left(\int_{\mathbb{R}^{3}} \rho(x)^{\frac{5}{3}} d x\right)^{\frac{1}{2}}\left(\int_{\mathbb{R}^{3}} \rho(x) d x\right)^{\frac{1}{2}} \\
& \leq \frac{1}{2}\left(\varepsilon \int_{\mathbb{R}^{3}} \rho(x)^{\frac{5}{3}} d x+\frac{1}{\varepsilon} \int_{\mathbb{R}^{3}} \rho(x) d x\right) .
\end{aligned}
$$

The estimates (A.5) and (A.6) with $\varepsilon=2 c_{\mathrm{LT}} / U c_{\mathrm{L}}$ prove the proposition.

\section{References}

[1] A.S. Alexandrov and N. Mott, Polarons and bipolarons, World Scientific, Singapore, 1996.

[2] Z. Ammari, Asymptotic completeness for a renormalized nonrelativistic Hamiltonian in quantum field theory: The Nelson model, Math. Phys. Anal. Geom. 3 (2000), 217-285.

[3] V. Bach, Error bound for the Hartree-Fock energy of atoms and molecules, Commun. Math. Phys. 147 (1992), 527-548.

[4] F. Brosens, S. N. Klimin, and J. T. Devreese, Variational path-integral treatment of a translation invariant many-polaron system, Phys. Rev. B 71 (2005), 214301, 13 pp.

[5] _ Path-integral approach to the ground-state energy of a homogeneous polaron gas, Phys. Rev. B 77 (2008), 085308, 9 pp.

[6] J. T. Cannon, Quantum field theoretic properties of a model of Nelson: Domain and eigenvector stability for perturbed linear operators, J. Funct. Anal. 8 (1971), 101-152.

[7] J. T. Devreese, Polarons, Encyclopedia of Applied Physics (G. L. Trigg and E. H. Immergut, eds.), vol. 14, Wiley-VCH, 1996, pp. 383-409.

[8] J. T. Devreese and J. Tempere, Large-polaron effects in the infrared spectrum of high- $T_{\mathrm{c}}$ cuprate superconductors, Solid State Commun. 106 (1998), 309-313.

[9] M. D. Donsker and S. R. Varadhan, Asymptotics for the polaron, Comm. Pure Appl. Math. (1983), 505-528.

[10] R. P. Feynman, Slow electrons in a polar crystal, Phys. Rev. 97, (1955), 660-665. 
[11] _ Statistical mechanics. A set of lectures, Frontiers in physics, W. A. Benjamin, Inc., Reading, Massechusets, 1972.

[12] H. Fröhlich, Electrons in lattice fields, Adv. in Phys. 3 (1954), 325-362.

[13] J. Fröhlich, Existence of dressed one-electron states in a class of persistent models, Fortschr. Phys. 22 (1974), 159-198.

[14] B. Gerlach and H. Löwen, Analytical properties of polaron systems or: Do polaronic phase transitions exist or not? , Rev. Modern Phys. 63 (1991), 63-90.

[15] M. Griesemer, Exponential decay and ionization thresholds in non-relativistic quantum electrodynamics, J. Funct. Anal. 210 (2004), 321-340.

[16] E. Haga, Note on the Slow Electrons in a Polar Crystal, Prog. Theoretical Phys. 11 (1954), 449-460.

[17] Ch. Hartinger, F. Mayr, J. Deisenhofer, A. Loidl, and T. Kopp, Large and small polaron excitations in La2/3(Sr/Ca)1/3MnO3 films, Phys. Rev. B 69 (2004), 100403, 4 pp.

[18] S. N. Klimin, V. M. Fomin, F. Brosens, and J. T. Devreese, Characterization of shellfilling of interacting polarons in a quantum dot through their optical absorption, Physica E 22 (2004), 494-497.

[19] T. D. Lee, F. E. Low, and D. Pines, The motion of slow electrons in a polar crystal, Phys. Rev. 90 (1953), 297-302.

[20] J.-M. Lévy-Leblond, Nonsaturation of gravitational forces, J. Math. Phys. 10 (1968), 806-812.

[21] E. H. Lieb, Existence and uniqueness of the minimizing solution of Choquard's nonlinear equation, Stud. Appl. Math. 70A (1977), 444-446.

[22] _ Thomas-Fermi and related theories of atoms and molecules, Rev. Mod. Phys. 53 (1981), 603-604, Erratum 54 (1981), 311.

[23] _ Variational principle for many-fermion systems, Phys. Rev. Lett. 46 (1981), 457-459, Erratum 47 (1981), 69.

[24] E. H. Lieb and M. Loss, Analysis, 2nd ed., Graduate Studies in Mathematics, vol. 14, American Mathematical Society, 2001.

[25] E. H. Lieb and L. E. Thomas, Exact ground state energy of the strong-coupling polaron, Comm. Math. Phys. 183 (1997), 511-519, Erratum 188 (1997) 499-500.

[26] T. Miyao and H. Spohn, The bipolaron in the strong coupling limit, Ann. Henri Poincaré 8 (2007), 1333-1370.

[27] E. Nelson, Interaction of non-relativistic particles with a quantized scalar field, J. Math. Phys. 5 (1964), 1190-1197. 
[28] G. Verbist, F. M. Peeters, and J. T. Devreese, Large bipolarons in two and three dimensions, Phys. Rev. B 43 (1991), 2712-2720. 